Technology in Cambridge and his colleagues designed gene circuits that act as 'kill switches' when they are introduced into the genome of the bacterium Escherichia coli. The switches block essential genes or induce lethal toxins when triggered by environmental chemicals.

Such kill switches can be customized to respond to different cues, the authors say. Nature Chem. Biol. http://dx.doi. org/10.1038/nchembio.1979 (2015)

\section{PLANT BIOTECHNOLOGY}

\section{CRISPR clips} crop genes

The CRISPR-Cas9 genomeediting system has been used to alter the genomes of two crop plants: barley (Hordeum vulgare; pictured) and Brassica oleracea, a species that includes broccoli and cabbage.

CRISPR-Cas9 allows researchers to easily engineer mutations in genomes and has been tested in some crops, including rice and wheat. Cristobal Uauy and Wendy Harwood at the John Innes Centre in Colney, UK, used the system in barley and the brassica species to knock out the function of genes encoding certain plant hormones that are involved in growth and seed development - both important agronomic traits.

The team generated heritable mutations and the modified plants contained no foreign genes. However, the editing system occasionally introduced unwanted, off-target genetic changes.

Genome Biol. 16, 258 (2015)

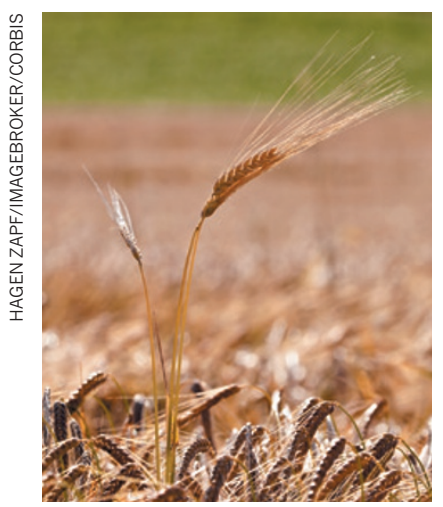

PHYSICS

\section{Long delay for electron decay}

An underground experiment has yielded the strongest evidence so far that electrons are stable, by showing that they last for at least 66 billion billion billion years before decaying into photons and neutrinos.

The Borexino detector, located underground at the Gran Sasso National Laboratory in central Italy, is looking mainly for neutrinos. The electron-stability result it produced is about 100 times longer than previous limits, and was obtained thanks to the extremely low natural radioactivity of the 300 tonnes of highly purified mineral oil at its core, according to Mauro Pallavicini of the University of Genoa, Italy, and his team.

Because the decay of negatively charged electrons into neutral particles seems even less likely than before, the results also confirm the fundamental law saying that net electric charge is conserved. In principle, however, electrons could still decay into some other unknown particles, the authors say.

Phys. Rev. Lett. 115, 231802

(2015)

\section{LASER PHYSICS}

\section{Lasers trigger $X$-rays efficiently}

Bombarding clouds of a noble gas with an intense UV laser emits X-ray pulses that could be useful for imaging applications.

Single atoms zapped by a UV laser emit bright X-rays, but to get such X-rays from a cloud of atoms, a longerwavelength laser is needed. To maximize the intensity of the emitted X-rays, Tenio Popmintchev at the University of Colorado Boulder and his colleagues found UV wavelengths that elicit strong emissions over a wide range of frequencies from single atoms or ions and clouds

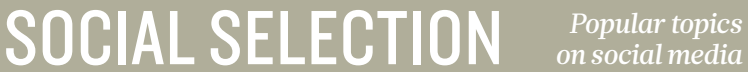

\title{
Should DNA donors see genomic data?
}

Geneticist Charles Danko turned to Twitter this week to ask for help in convincing his institutional review board (IRB) at Cornell University in Ithaca, New York, that he should be allowed to let his study participants download their own genetic information. He tweeted: "Writing IRB amendment to give subjects their genomic data. IRB does not like. Suggested reading to make the case?" Several scientists responded to Danko's request, including computational geneticist Yaniv Erlich at Columbia University in New York City, who pointed out that direct-to-consumer (DTC) genetics companies such as 23andMe already give people their data. "Another argument is

that $\sim 2$ million people have access to thei $\checkmark$ NATURE.COM raw genetic data through DTC," he wrote. For more on "No adverse events have been reported popular papers: $\quad$ so far due to access to raw data. Other go.nature.com/bxjd8q research projects do the same."

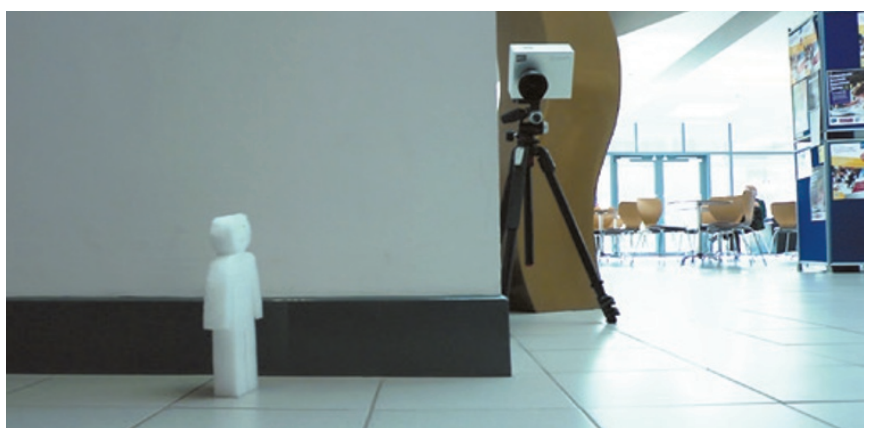

of these particles. By carefully choosing a laser's colour and shaping a guide for the light, the team created intense X-ray pulses just 100 attoseconds long ( 1 attosecond is $1 \times 10^{-18}$ seconds).

The technique produces light that could be powerful enough to probe the details of electron motion, the authors report.

Science 350, 1225-1231 (2015)

\section{PHOTONICS}

\section{Seeing movement around corners}

A camera can track the movement of an object hidden around a corner.

Researchers can already reveal images of hidden items by firing a laser onto the floor and studying the light that is scattered back from all surfaces, including concealed ones. But such systems work too slowly to track any movement. Genevieve Gariepy and Daniele Faccio at HeriotWatt University in Edinburgh, UK, and their colleagues used a laser and an ultrafast camera to measure the position and arrival time of photons from the laser bouncing back off a figurine placed around a corner (pictured). This allowed them to track the object, even while it was moving at a speed of a few centimetres per second.

The method could be useful for remote observation in hazardous areas, or to prevent vehicles colliding at blind corners, the authors say. Nature Photon. http://dx.doi.org/ 10.1038/nphoton.2015.234 (2015)

\section{$\rightarrow$ NATURE.COM}

For the latest research published by Naturevisit:

www.nature.com/latestresearch 